\title{
Oseltamivir prophylaxis for the prevention of influenza in healthy healthcare workers: Tolerability and compliance challenges
}

\author{
Westyn Branch-Elliman MD, MMSc ${ }^{1,2,3}$ (1), David S. Yassa MD, $\mathrm{MPH}^{2,4}$ and Sharon B. Wright MD, MPH ${ }^{2,4,5}$ \\ ${ }^{1}$ Section of Infectious Diseases Department of Medicine, Veterans' Affairs (VA) Boston Healthcare System, West Roxbury, Massachusetts, ${ }^{2} \mathrm{Harvard}$ Medical School, \\ Boston, Massachusetts, ${ }^{3}$ VA Boston Center for Healthcare Organization and Implementation Research (CHOIR), Boston, Massachusetts, ${ }^{4}$ Division of Infectious \\ Diseases, Beth Israel Deaconess Medical Center, Boston, Massachusetts and ${ }^{5}$ Division of Infection Control/Hospital Epidemiology, Silverman Institute for Health \\ Care Quality and Safety, Beth Israel Deaconess Medical Center, Boston, Massachusetts
}

To the Editor - We read with interest the important study by Sharpe et $\mathrm{al}^{1}$ about reasons why healthcare workers receive vaccination waivers. Influenza in healthcare personnel (HCP) can result in up to a week of paid or unpaid sick leave, causing substantial expense to a healthcare system and the healthcare worker. Staffing shortages attributable to influenza outbreaks are costly and limit the ability of hospitals to care for sick patients. The influenza vaccine is of limited efficacy and vaccine mismatches are not uncommon. These challenges create a critical need for alternative influenza prevention strategies for workforce and patient protection.

Several infection prevention strategies can be implemented to limit HCP exposure; commonly employed methods include the use of droplet precautions with either surgical masks or N95 respirators for HCP caring for known patients with influenza and pre-emptively masking all patients presenting with respiratory symptoms. ${ }^{2}$ Universal vaccination of $\mathrm{HCP}$ is another strategy that has been utilized to reduce influenza transmission to patients and has been widely adopted. ${ }^{3,4}$ However, despite the best infection prevention efforts, HCP are often exposed to influenza, either in the hospital before a case is recognized or in the community. Thus, the use of antivirals, such as oseltamivir, either for preemptive or postexposure prophylaxis is an alternative risk reduction strategy. ${ }^{5}$ However, little is known about the tolerability of oseltamivir prophylaxis in healthy HCP and about rates of compliance with treatment. Thus, we administered a survey during pandemic influenza seasons where oseltamivir prophylaxis was widely offered to quantify the incidence of side effects and medication discontinuation in HCP.

Any HCP who was exposed to influenza during 1 of 2 pandemic periods at a large tertiary-care academic medical center and who received oseltamivir for influenza prophylaxis through the hospital's employee health service was included in a cross-sectional survey. Pre-emptive prophylaxis was provided for workforce preservation during 1 year with high rates of vaccine failure and during another year before vaccine was available. Other prescriptions were for postexposure prophylaxis only. The survey included questions related to experienced side effects, days of oseltamivir completed, and the reason(s) for early discontinuation of prophylaxis (defined

Author for correspondence: Westyn Branch-Elliman, MD, 1400 VFW Parkway, West Roxbury, MA 02132. Email: wbranche@bidmc.harvard.edu

PREVIOUS PRESENTATION. This information was presented in part at the SHEA Annual Scientific Meeting on March 20, 2006, in Chicago, Illinois.

Cite this article: Branch-Elliman W, Yassa DS, and Wright SB. (2019). Oseltamivir prophylaxis for the prevention of influenza in healthy healthcare workers: Tolerability and compliance challenges. Infection Control \& Hospital Epidemiology, 40: 1328-1329, https://doi.org/10.1017/ice.2019.232
Table 1. Symptoms Reported by Number of Days Oseltamivir Taken

\begin{tabular}{lccc}
\hline Symptom & $\begin{array}{c}\text { Total } \\
(\mathrm{n}=138), \text { No. }(\%)\end{array}$ & $\begin{array}{c}1-9 \text { Days } \\
(\mathrm{n}=43), \text { No. }(\%)\end{array}$ & $\begin{array}{c}\geq 10 \text { Days } \\
(\mathrm{n}=95), \text { No. }(\%)\end{array}$ \\
\hline Abdominal pain & $17(12.3)$ & $10(23.3)$ & $7(7.4)$ \\
\hline Cough & $4(2.9)$ & $3(7.0)$ & $1(1.1)$ \\
\hline Diarrhea & $7(5.1)$ & $5(11.6)$ & $2(2.1)$ \\
\hline Dizziness & $4(2.9)$ & $3(7.0)$ & $1(1.1)$ \\
\hline Fatigue & $5(3.6)$ & $3(7.0)$ & $2(2.1)$ \\
\hline Nausea & $33(23.9)$ & $12(27.9)$ & $21(22.1)$ \\
\hline Headache & $7(5.1)$ & $3(7.0)$ & $4(4.2)$ \\
\hline Insomnia & $8(5.8)$ & $2(4.7)$ & $6(6.3)$ \\
\hline Rhinorrhea & $3(2.2)$ & $2(4.7)$ & $1(1.1)$ \\
\hline Sore throat & $3(2.2)$ & $2(4.7)$ & $1(1.1)$ \\
\hline Vomiting & $2(1.4)$ & $1(2.3)$ & $1(1.1)$ \\
\hline Other & $4(2.9)$ & $2(4.7)$ & $2(2.1)$ \\
\hline
\end{tabular}

as receipt of $<10$ days of therapy). Outreach to encourage survey completion was done via e-mail and phone.

In total, $226 \mathrm{HCP}$ were offered prophylaxis, and 144 accepted a course of oseltamivir. Of these 144, 142 completed the survey (98.6\%). Among responders to the survey, 95 of $142(66.9 \%)$ completed $\geq 10$ days of therapy, 43 of $142(30.3 \%)$ completed $1-9$ days of therapy, and 4 of 142 (2.81\%) requested postexposure prophylaxis but subsequently chose not to initiate it. The most common reason for discontinuation of therapy was adverse effects of the medication (34.8\%) followed by a perception of no longer being at risk for acquisition of influenza (eg, leaving for vacation or transitioning to another clinical service where patients were felt to be at lower risk of influenza). Furthermore, 22.8\% reported discontinuation because they forgot to continue taking the medication.

Rates of side effects stratified by total duration of treatment are presented in Table 1. A high proportion of participants in our study reported nausea (33 of 142, 23.9\%), abdominal pain (17 of 142, $12.3 \%$ ), insomnia (8 of $142,15.8 \%$ ), diarrhea (7 of $142,5.1 \%$ ), and headache (7 of 142, 5.9\%). For HCP who developed nausea and insomnia, a higher incidence was reported among those who discontinued treatment than among those who completed a full prophylactic course.

The use of antiviral medications, such as oseltamivir, to reduce nosocomial transmission of influenza in healthcare settings is a 
consideration during pandemic periods, particularly if an influenza vaccine is not available, if there is a poor vaccine match to the predominant circulating strain, or if HCP chose to waive vaccination for various reasons (as discussed by Sharpe et $\mathrm{al}^{1}$ ). Oseltamivir prophylaxis is well tolerated with minimal side effects according to surveys among household contacts of individuals diagnosed with influenza $^{6}$ and among nursing home residents. ${ }^{7}$ These studies report rates of nausea of $5.5 \%$ and $0.5 \%$ and discontinuation rates of $1.0 \%$ and $2.8 \%$ among household contacts and nursing home residents, respectively. Additional studies also report a very low incidence of side effects, even among individuals receiving high doses of the medication. ${ }^{8}$ However, in our survey of healthy $\mathrm{HCP}$, the use of oseltamivir prophylaxis was associated with substantially higher rates of nausea and other adverse medication effects. Another factor driving noncompliance may have been that $\mathrm{HCP}$ receiving pre-emptive prophylaxis did not perceive ongoing risk of contracting clinical illness. Thus, their focus on symptoms attributed to the antiviral medication outweighed concern about developing influenza when making decisions about whether to continue therapy and potential downsides of this approach to prevention. This finding may suggest that direct counseling about the ongoing risk of developing influenza after exposure should be emphasized because HCP may have misperceptions about the incubation period of influenza and the rationale for a more extended prophylaxis period to prevent clinical infection.

In summary, oseltamivir prophylaxis is a consideration in HCP populations at high risk of developing clinical disease, including in providers who decline vaccination for various reasons and in years with high rates of vaccination failure or delayed or limited vaccine availability. However, HCP may experience higher rates of adverse side effects when receiving oseltamivir than other populations. Thus, when considering institutional responses to influenza pandemics and implementation of oseltamivir for pre-emptive and postexposure prophylaxis, tolerability of oseltamivir is an important variable that may affect the utility and uptake of this intervention.

Acknowledgments. The authors thank Casey K. Mix-McNulty, Jessica Day, Robin Kalaidjian, and Linda Baldini for their contributions to this work.

Conflicts of interest. W.B.E. is supported by National Institutes of Health NHLBI 1K12HL138049-01. All other authors report no conflicts of interest relevant to this article.

Financial support. No financial support was provided relevant to this article.

\section{References}

1. Sharpe AR, LeClair-Netzel M, Wagner M, et al. Healthcare worker influenza vaccine waivers at an academic health system. Infect Control Hosp Epidemiol 2019;40:826-828.

2. Branch-Elliman W, Savor Price C, McGeer A, Perl TM. Protecting the frontline: designing an infection prevention platform for preventing emerging respiratory viral illnesses in healthcare personnel. Infect Control Hosp Epidemiol 2015;36:336-345.

3. Talbot TR, Babcock H, Caplan AL, et al. Revised SHEA position paper: influenza vaccination of healthcare personnel. Infect Control Hosp Epidemiol 2010;31:987-995.

4. Nichol KL, Lind A, Margolis KL, et al. The effectiveness of vaccination against influenza in healthy, working adults. N Engl J Med 1995;333:889-893.

5. Gorisek Miksic N, Ursic T, Simonovic Z, et al. Oseltamivir prophylaxis in controlling influenza outbreak in nursing homes: a comparison between three different approaches. Infection 2015;43:73-81.

6. Welliver R, Monto AS, Carewicz O, et al. Effectiveness of oseltamivir in preventing influenza in household contacts: a randomized controlled trial. JAMA 2001;285:748-754.

7. Bowles SK, Lee W, Simor AE, et al. Use of oseltamivir during influenza outbreaks in Ontario nursing homes, 1999-2000. J Am Geriatr Soc 2002; 50:608-616.

8. Dutkowski R, Smith JR, Davies BE. Safety and pharmacokinetics of oseltamivir at standard and high dosages. Int J Antimicrob Agents 2010;35:461-467.

\section{Possibility of nosocomial person-to-person transmission of hemorrhagic fever with renal syndrome}

\section{Jong Wook Park MD, Eun-Jeong Joo MD, PhD and Hae Suk Cheong MD, PhD (1)}

Division of Infectious Disease, Department of Internal Medicine, Kangbuk Samsung Hospital, Sungkyunkwan University School of Medicine, Seoul, Republic of Korea

To the Editor-Hantaviruses belong to the family Bunyaviridae and mainly infect small mammals. In humans, however, they cause febrile disease, usually named hemorrhagic fever with renal syndrome (HFRS) in Asia and Europe and hantavirus cardiopulmonary syndrome (HCPS) or hantavirus pulmonary syndrome (HPS) in the Americas. ${ }^{1,2}$

Hantavirus transmission from rodents to humans usually occurs via inhalation of aerosolized rodent urine, saliva, and feces, but rarely by rodent bites, ${ }^{1}$ and person-to-person transmission has

Author for correspondence: Hae Suk Cheong, E-mail: philliper@naver.com.

Cite this article: Park JW, Joo E-J, and Cheong HS. (2019). Possibility of nosocomial person-to-person transmission of hemorrhagic fever with renal syndrome. Infection Control \& Hospital Epidemiology, 40: 1329-1331, https://doi.org/10.1017/ice.2019.267 never been conclusively demonstrated. Among the genus Hantavirus, person-to-person transmission of Andes virus was documented in a physician who acquired the infection after exposure to patients infected with the Andes. ${ }^{3-5}$ However, 2 other studies have reported no evidence of person-to-person transmission of Andes virus. ${ }^{6,7}$ Furthermore, there is currently no evidence of person-to-person transmission of HFRS caused by Hantaan virus. Recently, a self-limited febrile illness was noted in several healthcare workers (HCWs) who had cared for a patient with HFRS in our hospital, raising concerns about the possibility of person-toperson transmission of Hantaan virus. In this study, we evaluated whether transmission of Hantaan virus had occurred among HCWs exposed to the patient with HFRS. This study was approved 